\title{
Enterobacter Related Implantable Cardiac Defibrillator Lead Endocarditis in Dialysis Patient
}

\author{
Hakan Duman, MD*, Oğuzhan Bodur, MD and Yüksel Çiçek, MD \\ Department of Cardiology, Faculty of Medicine, Recep Tayyip Erdoğan University, Turkey
}

*Corresponding author: Hakan Duman, MD, Associate Professor, Department of Cardiology, Faculty of Medicine, Recep Tayyip Erdoğan University, 53100 Rize, Turkey, Tel: +90-464-2130491, Fax: +90-464-2170364

\begin{abstract}
Use of intracardiac devices for heart diseases is increasing Worldwide. One of the important complications of pacemakers is infective endocarditis from the leads as the source. In this case, we report a 61-years-old male patient who is on dialysis and presented with implantable cardioverter defibrillator (ICD) lead endocarditis caused by Enterobacter Cloacae four months following implantation.
\end{abstract}

Learning objective: Intracardiac devices are one of the predisposing conditions for infective endocarditis. It is known that the immune system is weak in patients with chronic renal failure. Patients with dialysis should be careful for endocarditis following intracardiac pace implantation.

\section{Keywords}

Endocarditis, Dialysis patient, Defibrillator lead

\section{Introduction}

Cardiac device-related endocarditis (CDE) is a phenomenon for which incidence is on the rise; it presents difficult management challenges to the clinician. Diagnosing problems that require the implantation of cardiac modulators presents a problem to the clinician that is gradually increasing in frequency specifically, CDE (related to pacemakers [PMs] and implantable cardioverter defibrillators). It is not surprising that the insertion of a foreign body into the thorax along with at least one metal lead passing through a large blood vessel in direct contact with the heart could lead to an infection related to the presence of foreign bodies. On one hand, there is the patient who needs the implanted device, and the potential morbidity and mortality associated with its removal. On the other hand, there is the problem of a persistent infection usually acquired during insertion of an electrical device that is resistant to many antibiotics, has a high recurrence rate, and necessitates an extensive operation to remove the device if removal is delayed [1]. Infective endocarditis (IE) in chronic haemodialysis (HD) is significantly more common and causes greater morbidity and mortality than in the general population, being second only to cardiovascular disease as the leading cause of death in this group of immunocompromised patients [2]. Using the United States Renal Data System database, Abbott, et al. found an age-adjusted incidence ratio of IE in the HD population of 17.9 compared with the general population [3].

\section{Case Report}

A 61-years-old man, who was on dialysis with heart failure due to dilated cardiomyopathy and right ventricular single chamber implanted an intracardiac defibrillator (ICD) four months ago, had complaints of fever and dyspnea. He was diagnosed urinary tract infection and started empiric antibiotic treatment. The patient was receiving dialysis treatment with the help of permanent dialysis catheter. His complaints persisted despite treatment and he was admitted to infectious disease clinic and consulted with cardiology. Physical examination was normal except elevated body temperature (38.5 ${ }^{\circ} \mathrm{C}$ ) and sinus tachycardia (110 bpm). Laboratory values showed increased leukocyte count (WBC: 17.47 10^3/ $\mu \mathrm{L}$ ), C-reactive protein (CRP: $10.4 \mathrm{mg} / \mathrm{dL}$ ), erythrocyte sedimentation rate (ESR: $47 \mathrm{~mm} / \mathrm{h})$ and creatinine $(4.56$ $\mathrm{mg} / \mathrm{dL}$ ). Other routine laboratory values were within normal limits.

Transthoracic echocardiography (TTE) showed a mass image on the intracardiac defibrillator lead. Trans-

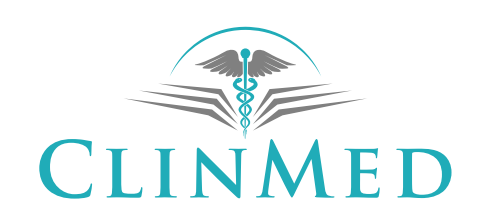

INTERNATIONAL LIBRARY

Citation: Duman H, Bodur O, Çiçek Y (2020) Enterobacter Related Implantable Cardiac Defibrillator Lead Endocarditis in Dialysis Patient. Int J Clin Cardiol 7:201. doi.org/10.23937/2378-2951/1410201

Accepted: September 14, 2020; Published: September 16, 2020

Copyright: (C) 2020 Duman $\mathrm{H}$, et al. This is an open-access article distributed under the terms of the Creative Commons Attribution License, which permits unrestricted use, distribution, and reproduction in any medium, provided the original author and source are credited. 


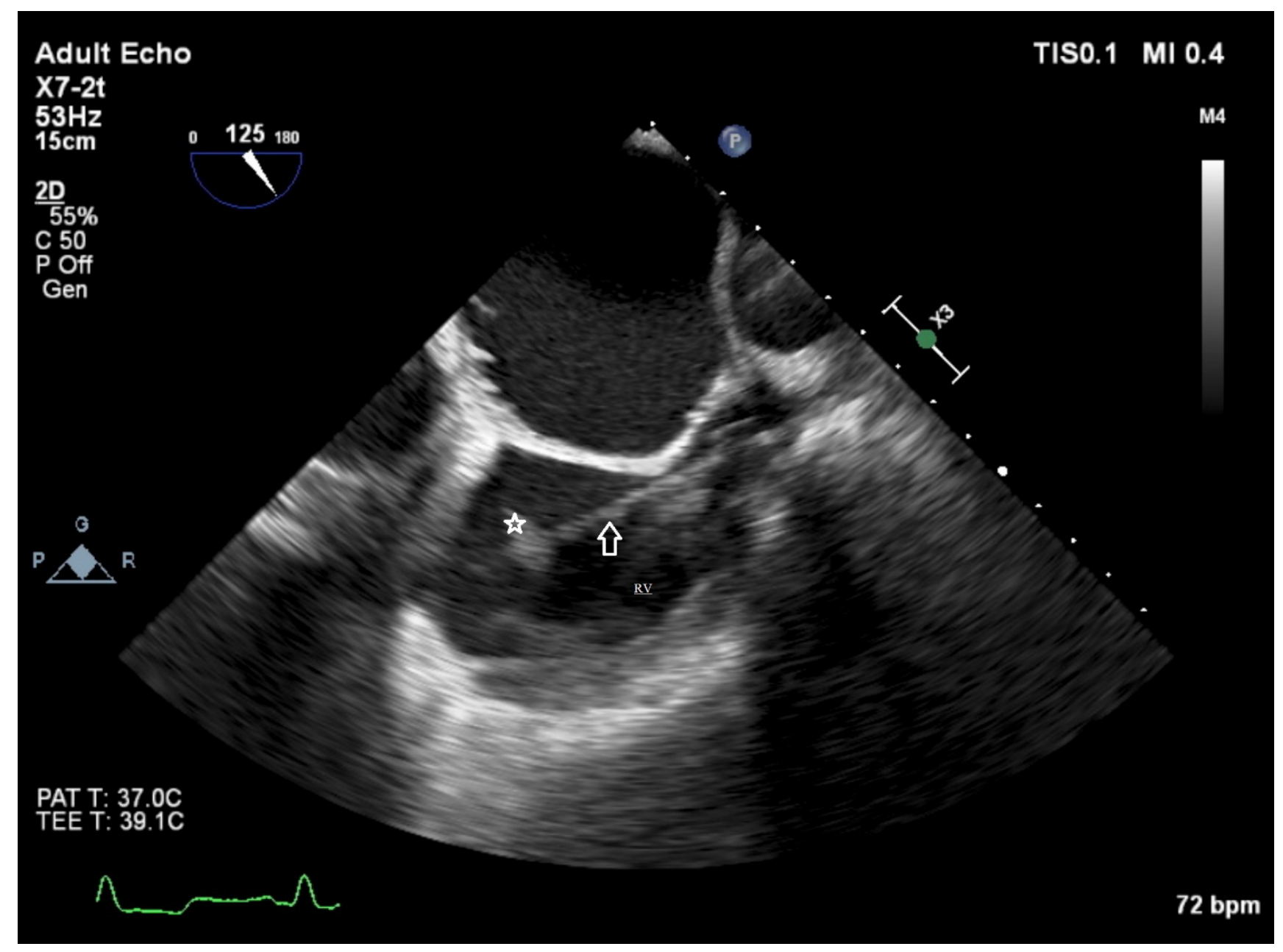

Figure 1: Transesophageal echocardiography shows a mass (vegetation) on the lead.

RV: Right Ventriculi; Arrow: Lead of the pace; Star: Mass (vegetation)

esophageal echocardiography (TEE) revealed, a mobile mass resembling a vegetation on the lead, unrelated the tricuspid valve, which was $9 \times 13 \mathrm{~mm}$ in diameter (Figure 1). The patient was hospitalized, three sets of blood cultures were drawn and vancomycin (500 mg once every three days) and meropenem (500 mg daily) were started. Blood cultures were positive for Enterobacter cloacae in two separate sets. Antibiogram showed that the microorganism was sensitive to the treatment protocol and the antibiotherapy was not changed. However, at the end of the second week of treatment, fever persisted and markers of inflammation (erythrocyte sedimentation rate [ESR] and C-reactive protein [CRP]) remained elevated. Thus, the patient was referred for transvenous lead extraction (TLE). Since the device was implanted in this patient within 1 year, lead was removed using a simple traction technique. And small vegatations on lead were detected. The culture of the extracted material was positive for Enterobacter cloacae. Antibiotherapy was appropriate according to the antibiogram and continued till the end of the postextracted sixth week.

\section{Discussion}

The incidence of septicemia and infective endocar- ditis reported in the literature after implantation of permanent pacemaker ranged from $0.5 \%$ to 7 [4]. Apart from general symptoms such as fever and malaise, peripheral clinical findings of infective endocarditis are not observed frequently in these patients; hence the diagnosis is especially hard, frequently delayed and sometimes even missed. The average time from symptom onset to diagnosis is reported 3-4 months. Risk factors for cardiac implantable electronic device infection can be divided into patient-related, procedural, and device-related factors. These risk factors may or may not be changed. Identifying modifiable risk factors is important as it can allow preventive measures to reduce risk. In patients with unchangeable risks, alternative approaches may be an option to reduce the overall risk. For example, kidney dialysis is an unchangeable patient risk factor [5]. Our patient was suffering from chronic renal failure and was receiving renal replacement therapy through the right subclavian catheter.

Lead endocarditis can be seen early or late after pacemaker implantation. Early endocarditis is defined as occurring within the first six months after implantation and causative microorganisms are most likely $S$. aureus and $S$. epidermidis [6]. The most common factors in the Cleveland Clinic series (87 pacemakers, 36 ICDs) 
were coagulase negative staphylococci (68\%), S. aureus (24\%) and enteric gram negative bacilli (17\%) [7]. Echocardiography is very important in the diagnosis of lead endocarditis. However, reviews suggest that TTE is inadequate. Victor, et al. evaluated 23 patients with lead endocarditis and found that TTE was capable of diagnosis in only $30 \%$ of patients, whereas TEE was able to diagnose $91 \%$ of the patient population [8]. Therefore, in patients with intracardiac defibrillator, evaluation of fever should always include a TEE examination. Extraction of contaminated material should be the cornerstone of therapy in patients with lead endocarditis [9]. Our case was also an example for this conclusion. Therefore, along with appropriate antibiotic treatment, the extraction of infected material is needed for therapy. The findings of the ELECTRa Registry demonstrate that despite successful TLE, mortality remains high for cardiovascular implantable electronic devices (CIED) infection. Furthermore, patients with infected CIED often have more severe long-standing comorbidities than other patients undergoing TLE. This highlights the need to reduce the incidence of CIED-related infections, especially in renal dialysis patients populations.Thus, one of the key lessons of the ELECTRa Registry may be that patients with device and lead infections require immediate attention and referral to a centre dealing with TLE [10].

In conclusion, patients with a permanent cardiac pacemaker with a risk factor for endocarditis should be investigated for endocarditis when they are admitted to the hospital due to fever. If endocarditis is detected, necessary antibiotherapy should be initiated and the permanent pacemaker should be extracted. With the advancement of technology, it is hoped that the use of subcutaneous devices, leedless devices and new antibiotic treatments will reduce the incidence of leed related endocarditis.

\section{Conflict of Interest}

The authors declare that there is no conflict of interest.

\section{References}

1. Edelstein S, Yahalom M (2009) Cardiac device-related endocarditis: Epidemiology, pathogenesis, diagnosis and treatment - A review. Int J Angiol 18: 167-172.

2. Hajsadeghi S, Pakbaz M, Aziz Ahari A, Kalantari S (2018) Co-infection with bacterial and fungal endocarditis at scar tissue in an immunocompromised patient. J Cardiol Cases 19: $117-120$

3. Abbott KC, Agodoa LY (2002) Hospitalizations for bacterial endocarditis after initiation of chronic dialysis in the United States. Nephron 91: 203-209.

4. Da Costa A, Kirkorian G, Chevalier P, Cerisier A, Chalvidan T, et al. (1998) Infections secondary to implantation of cardiac pacemakers. Arch Mal Coeur Vaiss 91: 753-757.

5. Blomström-Lundqvist C, Traykov V, Erba PA, Burri H, Nielsen JC, et al. (2020) European Heart Rhythm Association (EHRA) international consensus document on how to prevent, diagnose, and treat cardiac implantable electronic device infections-endorsed by the Heart Rhythm Society (HRS), the Asia Pacific Heart Rhythm Society (APHRS), the Latin American Heart Rhythm Society (LAHRS), International Society for Cardiovascular Infectious Diseases (ISCVID) and the European Society of Clinical Microbiology and Infectious Diseases (ESCMID) in collaboration with the European Association for Cardio-Thoracic Surgery (EACTS). Europace 22: 515-549.

6. G Bluhm, I Julander, M Levander-Lindgren, C Olin (1982) Septicemia and endocarditis--uncommon but serious complications in connection with permanent cardiac pacing. Scand J Thorac Cardiovasc Surg 16: 65-70.

7. Chua JD, Wilkof BL, Lee I, Juratli N, Longworth DL, et al. (2000) Diagnosis and management of infections involving implantable electrophyciologic cardiac devices. Ann Intern Med 133: 604-608.

8. Victor F, DePlace C, Camus C, Breton H Le, Leclercq C, et al. (1999) Pacemaker lead infection: Echocardiographic features, management, and outcome. Heart 81: 82-87.

9. Gilbert H, Patrizio L, Manuel JA, Maria GB, Jean-Paul C, et al. (2015) 2015 ESC Guidelines for the management of infective endocarditis: The Task Force for the Management of Infective Endocarditis of the European Society of Cardiology (ESC). Endorsed by: European Association for Cardio-Thoracic Surgery (EACTS), the European Association of Nuclear Medicine (EANM) Eur Heart J 36: 3075-3128.

10. Auricchio A, Regoli F, Conte G, Caputo ML (2017) Key lessons from the ELECTRa registry in the modern era of transvenous lead extraction. Arrhythm Electrophysiol Rev 6: 111-113.

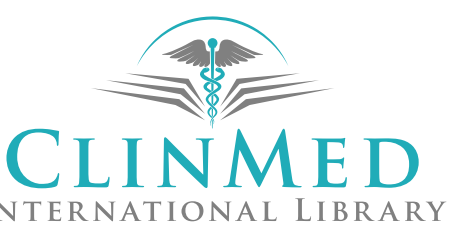

